\title{
Large-Eddy Simulation of the Temporal Mixing Layer Using the Clark Model
}

\author{
Bert Vreman, Bernard Geurts, and Hans Kuerten \\ Department of Applied Mathematics, University of Twente, \\ P.O. Box 217, 7500 AE Enschede, The Netherlands \\ Communicated by M.Y. Hussaini
}

Received 23 August 1994 and accepted 30 June 1995

\begin{abstract}
The Clark model for the turbulent stress tensor in large-eddy simulation is investigated from a theoretical and computational point of view. In order to be applicable to compressible turbulent flows, the Clark model has been reformulated. Actual large-eddy simulation of a weakly compressible, turbulent, temporal mixing layer shows that the eddy-viscosity part of the original Clark model gives rise to an excessive dissipation of energy in the transitional regime. On the other hand, the model gives rise to instabilities if the eddy-viscosity part is omitted and only the "gradient" part is retained. A linear stability analysis of the Burgers equation supplemented with the Clark model is performed in order to clarify the nature of the instability. It is shown that the growth-rate of the instability is infinite in the inviscid limit and that sufficient (eddy-)viscosity can stabilize the model. A model which avoids both the excessive dissipation of the original Clark model as well as the instability of the "gradient" part, is obtained when the dynamic procedure is applied to the Clark model. Large-eddy simulation using this new dynamic Clark model is found to yield satisfactory results when compared with a filtered direct numerical simulation. Compared with the standard dynamic eddy-viscosity model, the dynamic Clark model yields more accurate predictions, whereas compared with the dynamic mixed model the new model provides equal accuracy at a lower computational effort.
\end{abstract}

\section{Introduction}

Large-eddy simulation (LES) is a promising technique to simulate turbulent flows (Rogallo and Moin, 1984). In LES only the large scales are explicitly solved and for this purpose the Navier-Stokes equations are filtered. The filtering introduces new terms, the so-called subgrid terms representing the effect of the small scales, which have to be modeled in order to close the filtered equations. A number of subgrid models for the most important subgrid term, the turbulent stress tensor, exist. Using an eddy-viscosity hypothesis is the most common procedure to model this tensor and the Smagorinsky model is the best-known example (Smagorinsky, 1963). Recently, a dynamic formulation of this model has been proposed (Germano, 1992), in which the model coefficient is dynamically adjusted to the local structure of the flow. This procedure considerably improves the performance of the eddy-viscosity model in transitional and inhomogeneous flows (Germano et al., 1991). However, the eddy-viscosity concept is not the only strategy which has been followed to model the turbulent stress tensor. Alternative formulations are provided by, e.g., the similarity model (Bardina et al., 1984) and the Clark model (Clark et al., 1979). An increasing amount of research is directed toward the use of the similarity idea in LES. The validity of this idea has been investigated using experimental data (Liu et al., 1994). Furthermore, LES has been conducted using the mixed model, in which the similarity model is supplemented with the Smagorinsky eddy-viscosity (Erlebacher et al., 1992). The 
dynamical formulation of the mixed model has also been adopted (Zang et al., 1993; Vreman et al., 1994b) and improvement over the standard dynamic eddy-viscosity model was observed. Compared with other subgrid models the dynamic mixed model produces good results, but a simulation using this model is quite expensive, because many explicit filtering operations are needed in each time step.

The model developed by Clark et al. (1979), which is the subject of this paper, has received less attention than the similarity model, although a priori tests of this model yield correlations which are at least as good as those of the similarity model (Clark et al., 1979; Vreman et al., 1993). The Clark model consists of two parts; the first part, which is based on Taylor expansions of the velocity field, contains inner products of velocity gradients and is called the "gradient" model, and the other part is the Smagorinsky eddy-viscosity model. Furthermore, the model satisfies several basic requirements, such as Galilean invariance (Speziale, 1985) and realizability (Vreman et al., 1994a). However, actual simulations using the Clark model are rarely found in the literature. One of the examples is the work of Love (1980) on the one-dimensional Burgers equation. In the present paper we present results of LES with the Clark model for the temporal mixing layer at low Mach number. First, in Section 2 we demonstrate that the derivation of the Clark model for the turbulent stress tensor in incompressible flow can be extended to compressible flow. Next, in Section 3 results of LES with the original Clark model show that the model is too dissipative in the transitional regime. However, if the eddy-viscosity part of the model, which is responsible for the excessive dissipation, is omitted, the simulation becomes unstable. An analysis of the nature of this instability for the onedimensional Burgers equation is presented in Section 4, which shows that the instability can be removed if a sufficient amount of dissipation is added. For this reason the dynamic Clark model is formulated in Section 5, which overcomes the problems of both excessive dissipation and instability. Finally, in Section 6 we summarize our conclusions.

\section{Closure of the Filtered Equations with the Clark Model}

We first present the filtered Navier-Stokes equations for a compressible flow and then demonstrate how the Clark model can be derived for the turbulent stress tensor using Taylor expansions.

The partial differential equations which govern a compressible flow are the Navier-Stokes equations, representing conservation of mass, momentum, and energy. In the LES of turbulent flows these equations are filtered in order to reduce the amount of scales to be solved. The filter operation extracts the large scale part $\bar{f}$ from a flow variable $f$ as follows:

$$
\bar{f}(\mathbf{x})=\int_{\Omega} G_{\Delta}(\mathbf{x}-\mathbf{y}) f(\mathbf{y}) d \mathbf{y} .
$$

Here $\Omega$ is the flow domain and $\Delta$ denotes the "filter width" associated with the kernel $G_{\Delta}$. We adopt a filter width which does not depend on the postion vector $\mathbf{x}$ and, consequently, the filter operation is a convolution and commutes with spatial derivatives (Rogallo and Moin, 1984). For compressible flows, Favre (1983) has introduced a related filter operation,

$$
\tilde{f}=\frac{\overline{\rho f}}{\bar{\rho}},
$$

where $\rho$ denotes the fluid density. The filtered Navier-Stokes equations can be written in the following form (Vreman et al., 1994c):

$$
\begin{aligned}
\partial_{t} \bar{\rho}+\partial_{j}\left(\bar{\rho} \tilde{u}_{j}\right) & =0, \\
\partial_{t}\left(\bar{\rho} \tilde{u}_{i}\right)+\partial_{j}\left(\bar{\rho} \tilde{u}_{i} \tilde{u}_{j}\right)+\partial_{i} \bar{p}-\partial_{j} \check{\sigma}_{i j} & =-\partial_{j}\left(\bar{\rho} \tau_{i j}\right), \\
\partial_{t} \check{e}+\partial_{j}\left((\check{e}+\bar{p}) \tilde{u}_{j}\right)-\partial_{j}\left(\check{\sigma}_{i j} \tilde{u}_{i}\right)+\partial_{j} \check{q}_{j} & =R,
\end{aligned}
$$

where the symbols $\partial_{t}$ and $\partial_{j}$ denote the partial differential operators $\partial / \partial t$ and $\partial / \partial x_{j}$, respectively. Furthermore, the summation convention for repeated indices is used. The independent variables $t$ and $x_{j}$ represent time and the spatial coordinates, respectively. 
Concerning the flow variables, the Favre-filtered velocity is denoted by $\tilde{\mathbf{u}}$, while $\bar{\rho}$ is the filtered density and $\bar{p}$ is the filtered pressure. Moreover, $\check{e}$ is the total energy density of the filtered variables

$$
\check{e}=\frac{\bar{p}}{\gamma-1}+\frac{1}{2} \bar{\rho} \tilde{u}_{i} \tilde{u}_{i}
$$

The viscous stress tensor based on filtered variables, $\breve{\sigma}_{i j}$, is defined as

$$
\check{\sigma}_{i j}=\frac{\mu(\tilde{T})}{R e} S_{i j}(\tilde{\mathbf{u}}) \quad \text { with } \quad S_{i j}(\tilde{\mathbf{u}})=\left(\partial_{j} \tilde{u}_{i}+\partial_{i} \tilde{u}_{j}-\frac{2}{3} \delta_{i j} \partial_{k} \tilde{u}_{k}\right)
$$

where $\delta_{i j}$ is the Kronecker delta and $\mu(\widetilde{T})$ is the dynamic viscosity, expressed by Sutherland's law for air,

$$
\mu(\widetilde{T})=\widetilde{T}^{3 / 2} \frac{1+C}{\tilde{T}+C}
$$

with $C=0.4$. In addition $\breve{\mathbf{q}}$ represents the heat-flux vector based on filtered variables, given by

$$
\check{q}_{j}=-\frac{\mu(\widetilde{T})}{(\gamma-1) \operatorname{Re} \operatorname{Pr} M_{\mathrm{R}}^{2}} \partial_{j} \tilde{T} .
$$

The Favre-filtered temperature $\widetilde{T}$ is related to the filtered density and the filtered pressure by the ideal gas law

$$
\tilde{T}=\gamma M_{\mathrm{R}}^{2} \frac{\bar{p}}{\bar{\rho}}
$$

These equations have been made dimensionless by introducing a reference length $L_{\mathrm{R}}$, velocity $u_{\mathrm{R}}$, density $\rho_{\mathrm{R}}$, temperature $T_{\mathrm{R}}$, and viscosity $\mu_{\mathrm{R}}$. In addition $\gamma$, the ratio of the specific heats $C_{P}$ and $C_{V}$, and the Prandtl number $\operatorname{Pr}$ are given the values $\gamma=1.4$ and $P r=1$. The values of the Reynolds number $R e=\rho_{\mathrm{R}} u_{\mathrm{R}} L_{\mathrm{R}} / \mu_{\mathrm{R}}$ and the reference Mach number $M_{\mathrm{R}}=u_{\mathrm{R}} / \sqrt{\gamma R_{\mathrm{g}} T_{\mathrm{R}}}$, where $R_{\mathrm{g}}$ is the universal gas constant, are given below.

In this description the left-hand sides of (3)-(5) are expressed in the filtered variables $\bar{\rho}, \tilde{u}_{j}$, and $\vec{p}$. The right-hand sides of the filtered equations contain the so-called subgrid terms. The subgrid term in the momentum equation (4) contains the turbulent stress tensor, defined as

$$
\bar{\rho} \tau_{i j}=\bar{\rho} u_{i} u_{j}-\frac{\overline{\rho u_{i}} \overline{\rho u}}{\bar{\rho}}=\bar{\rho}\left({\widetilde{u_{i}}}_{j}-\tilde{u}_{i} \tilde{u}_{j}\right) .
$$

The effects of the subgrid terms in the energy equation (5) are negligible for the weakly compressible mixing layer considered in this paper (Vreman et al., 1994c). Terms created by nonlinearities in the viscous stress tensor can also be neglected.

In the following we derive the Clark model for $\vec{\rho} \tau_{i j}$ using Taylor expansions of the filtered velocity. Our procedure is slightly different from the procedure followed by Clark et al. (1979), since we do not apply the expansions to the Leonard, cross, and Reynolds components separately, but to the turbulent stress itself. Furthermore, we present the formulation for the compressible turbulent stress tensor, thus generalizing the derivation by Clark et al. The same technique can also be applied in the construction of models for other subgrid terms, for example, the pressure-velocity or pressure dilation subgrid term (Vreman et al., 1994c). The filter that we use is the top-hat filter, which is defined by its kernel:

$$
G_{\Delta}(\mathbf{y})= \begin{cases}\frac{1}{\Delta_{1} \Delta_{2} \Delta_{3}} & \text { if } \quad\left|y_{i}\right|<\frac{\Delta_{i}}{2} \quad(i=1,2,3), \\ 0 & \text { otherwise, }\end{cases}
$$

where $\Delta_{i}$ is the filter width in the $i$-direction and $\Delta=\left(\Delta_{1} \Delta_{2} \Delta_{3}\right)^{1 / 3}$. Filters commonly used in LES are the top-hat, the Gaussian, and the spectral cut-off filter. Vreman et al. (1994a) argue that from the viewpoint of realizability the Clark model should be used in conjunction with positive filters, e.g., the top-hat or Gaussian filter. In this work we have selected the top-hat filter, since it is more attractive than the Gaussian 
filter with respect to computational cost. For the top-hat filter $\bar{f}$ is defined as

$$
\bar{f}(\mathbf{x})=\frac{1}{\Delta_{1} \Delta_{2} \Delta_{3}} \int_{-\Delta_{3} / 2}^{\Delta_{3} / 2} \int_{-\Delta_{2} / 2}^{\Delta_{2} / 2} \int_{-\Delta_{\Delta} / 2}^{\Delta_{1} / 2} f(\mathbf{x}+\mathbf{y}) d \mathbf{y} .
$$

The function $f(\mathbf{x}+\mathbf{y})$ is expanded as a Taylor series around $\mathbf{x}$, and after evaluation of the integral we obtain

$$
\bar{f}=f+\frac{1}{24} \Delta_{k}^{2} \partial_{k}^{2} f+O\left(\Delta^{4}\right) .
$$

The same formula holds for Gaussian filters (Love, 1980). We use this formula to rewrite the turbulent stress tensor:

$$
\begin{aligned}
\bar{\rho} \tau_{i j} & =\overline{\rho u_{i} u_{j}}-\frac{\overline{\rho u_{i}} \rho \bar{u}_{j}}{\bar{\rho}} \\
& =\rho u_{i} u_{j}+\frac{1}{24} \Delta_{k}^{2} \partial_{k}^{2}\left(\rho u_{i} u_{j}\right)-\frac{\left(\rho u_{i}+\frac{1}{24} \Delta_{k}^{2} \partial_{k}^{2}\left(\rho u_{i}\right)\right)\left(\rho u_{j}+\frac{1}{24} \Delta_{k}^{2} \partial_{k}^{2}\left(\rho u_{j}\right)\right)}{\rho+\frac{1}{24} \Delta_{k}^{2} \partial_{k}^{2} \rho}+O\left(\Delta^{4}\right) \\
& =\frac{1}{12} \Delta_{k}^{2} \rho\left(\partial_{k} u_{i}\right)\left(\partial_{k} u_{j}\right)+O\left(\Delta^{4}\right),
\end{aligned}
$$

where we used the relation

$$
\frac{1}{\rho+\frac{1}{24} \Delta_{k}^{2} \partial_{k}^{2} \rho}=\frac{1}{\rho}-\frac{1}{24 \rho^{2}} \Delta_{k}^{2} \partial_{k}^{2} \rho+O\left(\Delta^{4}\right)
$$

The next step is to express (15) into filtered variables. Using (16), the Favre-filtered velocity can be written as

$$
\begin{aligned}
\tilde{u}_{i} & =\frac{\overline{\rho u}_{i}}{\bar{\rho}} \\
& =\frac{\rho u_{i}+\frac{1}{24} \Delta_{k}^{2} \partial_{k}^{2}\left(\rho u_{i}\right)}{\rho+\frac{1}{24} \Delta_{k}^{2} \partial_{k}^{2} \rho}+O\left(\Delta^{4}\right) \\
& =u_{i}+\frac{1}{24} \Delta_{k}^{2} \partial_{k}^{2} u_{i}+\frac{1}{12 \rho} \Delta_{k}^{2}\left(\hat{\partial}_{k} \rho\right)\left(\hat{o}_{k} u_{i}\right)+O\left(\Delta^{4}\right) .
\end{aligned}
$$

We observe that for both the bar and Favre filter, unfiltered and filtered variables differ by a term of the order $O\left(\Delta^{2}\right)$ :

$$
\begin{aligned}
\rho & =\tilde{\rho}+O\left(\Delta^{2}\right), \\
u_{i} & =\tilde{u}_{i}+O\left(\Delta^{2}\right) .
\end{aligned}
$$

Substituting expressions (18) and (19) into (15) yields

$$
\bar{\rho} \tau_{i j}=\frac{1}{12} \Delta_{k}^{2} \bar{\rho}\left(\partial_{k} \tilde{u}_{i}\right)\left(\partial_{k} \tilde{u}_{j}\right)+O\left(\Delta^{4}\right)
$$

The first term on the right-hand side is referred to as the "gradient" model. Observe that this expansion is mathematically correct provided the variables can be differentiated sufficiently often. However, for rapidly fluctuating variables, the $O\left(\Delta^{4}\right)$ term may not be small and hence simply omitting the rest-term can be inaccurate. Clark et al. have inserted the Smagorinsky eddy-viscosity model for this rest-term, which finally yields the original Clark model:

$$
\bar{\rho} \tau_{i j}=\frac{1}{12} \Delta_{k}^{2} \bar{\rho}\left(\hat{\partial}_{k} \tilde{u}_{i}\right)\left(\partial_{k} \tilde{u}_{j}\right)-C_{\mathrm{S}}^{2} \Delta^{2} \bar{\rho}|S(\tilde{\mathbf{u}})| S_{i j}(\tilde{\mathbf{u}}),
$$

where $|S(\tilde{\mathbf{u}})|^{2}=\frac{1}{2} S_{i j}^{2}(\tilde{\mathbf{u}})$ and the model coefficient $C_{\mathrm{S}}=0.17$, a value suggested by Clark et al. (1979) and Schumann (1991). Equations (18) and (19) show that the Smagorinsky model is $O\left(\Delta^{2}\right)$, whereas according to (20) a model for the rest-term is preferred to be $O\left(\Delta^{4}\right)$. At present, however, no satisfactory $O\left(\Delta^{4}\right)$ model is available, therefore we consider the Smagorinsky model, which is of the order $O\left(\Delta^{2}\right)$, for the rest-term.

The Clark et al. model has been called "mathematically indefensible" by Love (1980) (compare the work by Rogallo and Moin (1984)), since Clark et al. used Taylor expansions of the subgrid-scale velocity field $u_{i}^{\prime}=u_{i}-\bar{u}_{i}$, which is not smoothly varying over lengths of $O(\Delta)$. The derivation in this paper is different from 
the derivation by Clark et al., since we do not use Taylor expansions of the subgrid-scale velocity field $u_{i}^{\prime}$, but Taylor expansions of the unfiltered variables $\rho$ and $u_{i}$. However, like the subgrid-scale quantities the unfiltered variables are not smoothly varying over lengths of $O(\Delta)$ either. This point does not imply that the expansion is mathematically incorrect (assuming that derivatives exist), although the higher-order terms may not be small compared with the terms retained. An alternative procedure is to use Taylor expansions of filtered variables only, which vary more smoothly over lengths of $O(\Delta)$. This can be achieved by considering not the turbulent stress itself, but the similarity model for the turbulent stress (Vreman et al., 1994d):

$$
\bar{\rho} \tau_{i j}^{\operatorname{sim}}=\overline{\bar{\rho} \tilde{u}_{i} \tilde{u}_{j}}-\frac{\overline{\bar{\rho} \tilde{u}_{i}} \overline{\bar{\rho} \tilde{u}_{j}}}{\overline{\bar{\rho}}}
$$

which is the definition of the turbulent stress in compressible flow applied to filtered variables. For incompressible flows this model reduces to the sum of the Leonard term and Bardina's similarity model for the cross-term (Bardina et al., 1984). By analogy to (15) the tensor given in (22) can be rewritten as

$$
\bar{\rho} \tau_{i j}^{\mathrm{sim}}=\frac{1}{12} \Delta_{k}^{2} \bar{\rho}\left(\partial_{k} \tilde{u}_{i}\right)\left(\partial_{k} \tilde{u}_{j}\right)+O\left(\Delta^{4}\right) .
$$

In this derivation only Taylor expansions of the filtered quantities $\bar{\rho}$ and $\tilde{u}_{i}$ are employed, whereas in (15) Taylor expansions of unfiltered variables are used. The expressions in (20) and (23) are identical, but the $O\left(\Delta^{4}\right)$ term in the latter case will in general be smaller. From this point of view the Smagorinsky eddy-viscosity in the Clark model accounts not only for the relatively small $O\left(\Delta^{4}\right)$ term in (23), but also for the discrepancy between the similarity model $\bar{\rho} \tau_{i j}^{\text {sim }}$ and the turbulent stress $\bar{\rho} \tau_{i j}$.

\section{Shortcomings of the Clark Model in Actual LES}

In this section we present results of LES of the temporal mixing layer using the Clark subgrid model. The results are compared with the results of a filtered direct numerical simulation (DNS). We first discuss the DNS, in which the unfiltered Navier-Stokes equations are solved.

In order to simulate a three-dimensional temporal mixing layer we solve the flow equations in a cubic geometry $[0, L] \times\left[-\frac{1}{2} L, \frac{1}{2} L\right] \times[0, L]$. Periodic boundary conditions are imposed in the $x_{1}-$ and $x_{3^{-}}$ direction, while in the $x_{2}$-direction the boundaries are free slip walls, i.e., the normal velocity and the normal derivatives of the density, pressure, and tangential velocities are zero. The basic initial velocity profile is the hyperbolic tangent profile $u=\tanh x_{2}$. The initial temperature profile is obtained from the BusemannCrocco law (Ragab and $\mathrm{Wu}, 1989$ ) and the initial pressure distribution is uniform. The reference length $L_{\mathrm{R}}$ is half the initial vorticity thickness, while the reference density, velocity, temperature, and dynamic viscosity are the initial upper stream values. As in other simulations of the three-dimensional mixing layer (Moser and Rogers, 1993; Comte et al., 1992), the length $L$ of the domain is set to four times the wavelength of the most unstable mode according to linear stability theory. We superimpose a three-dimensional large amplitude perturbation on the mean profile, as described by Sandham and Reynolds (1991). The amplitudes of the disturbances are 0.05 for the two-dimensional and 0.15 for the oblique instability waves. In this paper we do not study compressibility effects, so a low convective Mach number $M_{\mathrm{R}}=0.2$ is used (Vreman et al., 1994c), while $R e=50$, following Comte et al. (1992).

The DNS database has been obtained by solving the Navier-Stokes equations, without any subgrid model, on a fine grid with $192^{3}$ cells. The numerical method employs a second-order accurate four-stage Runge-Kutta method for marching in time (Vreman et al., 1992), whereas the spatial derivatives are discretized using a fourth-order scheme for the convective and a second-order scheme for the viscous terms (Vreman et al., 1994c). A higher-order scheme for the marching in time is not required, since the trunctation errors introduced by the discretization of the time derivatives are considerably smaller than the truncation errors introduced by the spatial discretizations. Visualization of the DNS demonstrates the roll-up of the fundamental instability and successive pairings. Four rollers with mainly negative spanwise voriticity are observed at $t=20$. After the first pairing ( $t=40$ ) the flow has become highly three-dimensional. Another pairing $(t=80)$ yields a single roller in which the flow exhibits a complex structure, with many regions of positive spanwise vorticity (Vreman et al., 1993). This structure is an effect of the transition to turbulence which has been triggered by the pairing process at $t=40$ (Moser and Rogers, 1993). The simulations are stopped at $t=100$, since the single roller at $t=80$ cannot undergo another pairing. The accuracy of the 


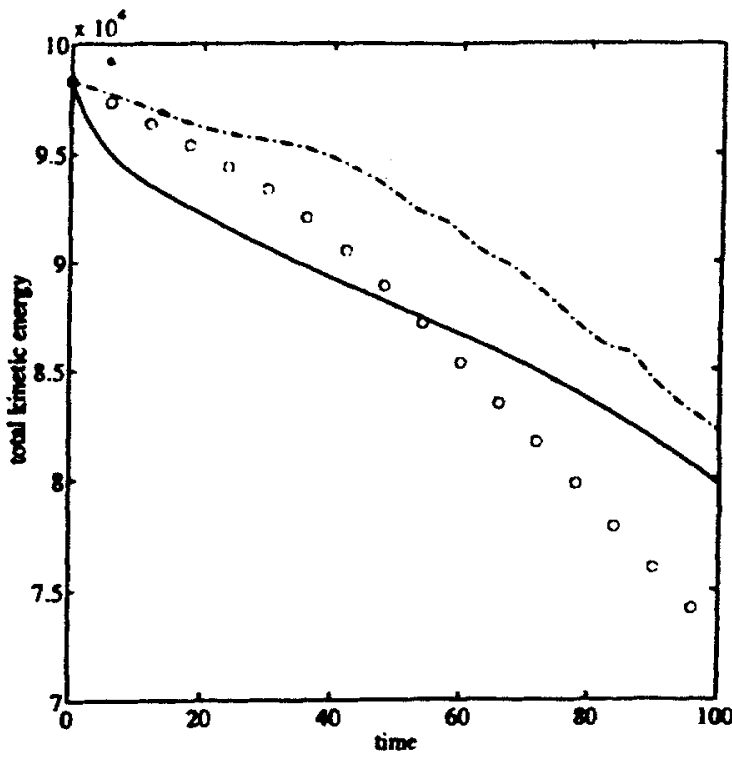

(a)

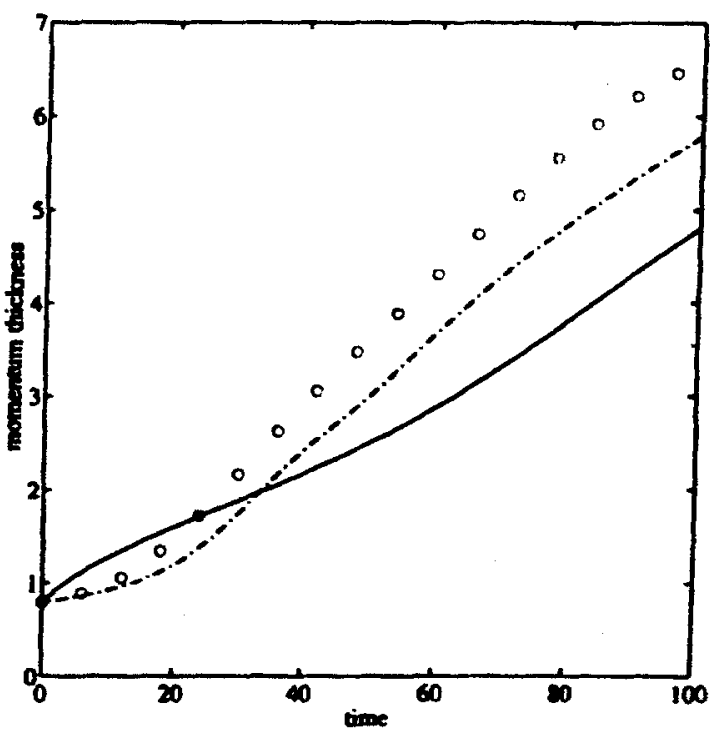

(b)

Figure 1. Results of LES with the Clark model (solid), the filtered DNS (circles), and LES without a subgrid-model (dashed-dotted). The evolution of the total kinetic energy (a) and the evolution of the momentum thickness (b) are shown.

simulation was found to be satisfactory. First, the linear growth rates of the dominant instability modes were captured within $1 \%$. Furthermore, in a simulation on a coarser grid $\left(128^{3}\right.$ cells) the results for the kinetic energy and momentum thickness were identical within plotting accuracy, whereas deviations in local quantities (e.g., slices through the vorticity field in the turbulent regime) were small.

The LES presented in this section have been performed on a grid with $32^{3}$ cells, which is considerably coarser than the DNS grid, and a filter width $\Delta=L / 16$ is used. Thus the filter width $\Delta$ equals twice the grid spacing of the coarse grid, which is an optimal choice (Vreman et al., 1994d). The simulation is performed with the same numerical method as the DNS. In order to compare the LES and DNS results the DNS results are filtered on the fine grid. Next, the filtered data is easily obtained on the coarse grid through restriction of the filtered fine-grid data. This procedure in particular provides the initial conditions of the LES.

Figure 1 shows the total kinetic energy $E$ and the momentum thickness $d$ calculated from the LES with the Clark model. The quantities $E$ and $d$ are defined as

$$
\begin{gathered}
E=\int_{\Omega} \frac{1}{2} \bar{\rho} \tilde{u}_{i} \tilde{u}_{i} d \mathbf{x}, \\
d=\int_{-L / 2}^{L / 2}\langle\bar{\rho}\rangle\left(1-\left\langle\tilde{u}_{1}\right\rangle\right)\left(1+\left\langle\tilde{u}_{1}\right\rangle\right) d x_{2},
\end{gathered}
$$

where $\langle\cdot\rangle$ denotes averaging over the homogeneous $x_{1}$ - and $x_{3}$-directions. The curves corresponding to the filtered fine-grid DNS and LES without incorporating a subgrid model ("coarse-grid DNS") have been included in Figure 1. Comparison with the fine-grid DNS results indicates that the behavior of the subgrid model is poor; LES without a subgrid model is better than LES with the Clark model. More specifically, the subgrid model is too dissipative: the decay of the total kinetic energy and the growth of the momentum thickness in the initial stages are too large. This behavior is caused by the Smagorinsky part of the Clark model. Indeed, the pure Smagorinsky model also exhibits an excessive dissipation in the transitional regime (Piomelli et al., 1990: Vreman et al., 1994d).

Since the Clark model is too dissipative in the transitional regime, we also perform a simulation using the gradient model, i.e., omitting the eddy-viscosity part in the Clark model. Figure 2 shows the evolution of the total kinetic energy of an LES with this subgrid model. The behavior in the transitional regime is more 
Figure 2. The evolution of the total kinetic energy for LES with the gradient model (solid), the filtered DNS (circles), and LES without a subgrid-model (dashed-dotted).

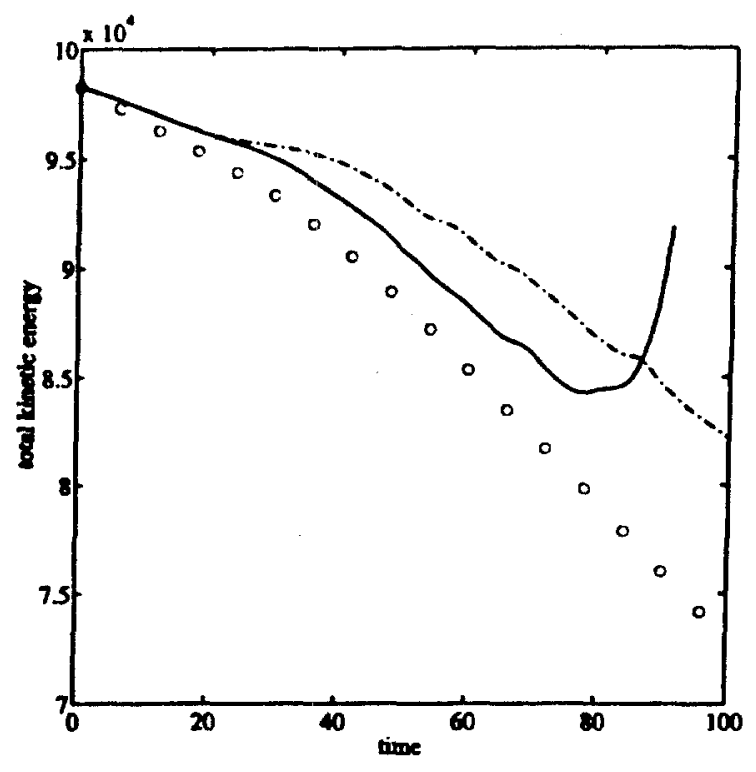

accurate than LES without a subgrid model. However, the simulation becomes unstable, which is illustrated by the strong increase of the total kinetic energy after $t=70$. The reason for this instability cannot simply be that the grid is too coarse, because the coarse-grid DNS which employs the same grid remains stable. Furthermore, similar to findings for the similarity model (22) (Vreman et al., 1994d), the gradient model can dissipate energy, since until $t=70$ the decay of energy is stronger than in the coarse-grid DNS. In a priori tests the gradient model gives correlations which are as high or even higher than those of the similarity model (Vreman et al., 1993). However, a posteriori tests show that although both models are able to dissipate energy, the gradient model causes stability problems, whereas the similarity model gives a stable simulation with acceptable results (Vreman et al., 1994d).

Summarizing we have the following shortcomings of the Clark model: if the eddy-viscosity in incorporated, it is excessively dissipative in the transitional regime, whereas without the eddy-viscosity the LES is unstable. In the next section we analyze the nature of this instability for a one-dimensional model problem. Then in Section 5 a new version of the Clark model is presented, resulting in a simulation which is both stable and considerably more accurate than the original Clark model.

\section{Instabilities in the Burgers Equation Using the Gradient Subgrid Model}

It is well known that the Burgers equation is a simple model that describes flow phenomena which are in several respects qualitatively similar to Navier-Stokes flows. Since the Burgers equation is a onedimensional scalar equation, mathematical analysis is often possible. The equation has been the subject of several studies of LES (Love, 1980; Humi, 1990). In this section we examine the Burgers equation supplemented with the one-dimensional version of the gradient part of the Clark model. The linear stability of a sinusoidal profile is investigated, in order to gain some understanding of the instability of the gradient model encountered in the previous section. The connection between linear and nonlinear stability is known for Navier-Stokes flows and has been formulated in the following way (Henningson and Reddy, 1994). If a flow is linearly unstable, then it is nonlinearly unstable to arbitrarily small initial disturbances. On the other hand, if a flow is linearly stable, then it is nonlinearly stable, provided the initial disturbance is sufficiently small. Linear analysis thus provides information on the nonlinear equation.

The Burgers equation with the gradient subgrid model is written as

$$
\partial_{t} u+\frac{1}{2} \partial_{x}\left(u^{2}\right)-v \partial_{x}^{2} u=-\frac{1}{2} \eta \partial_{x}\left(\partial_{x} u\right)^{2}+f(x),
$$

where $\partial_{t}$ and $\partial_{x}$ denote the time and spatial derivative, respectively, $u$ is the one-dimensional velocity, and 
$v$ is the viscosity. The left-hand side of this equation contains all terms in the standard Burgers equation. The right-hand side represents the gradient model with parameter $\eta=\frac{1}{2} \Delta^{2}$ plus a forcing term $f$.

The following analysis shows that smooth solutions of (26) can be extremely sensitive to small perturbations, leading to severe instabilities. In particular, we consider the linear stability of a $2 \pi$-periodic stationary solution, $U(x, t)=\sin (x)$, on the domain $[0,2 \pi]$ with periodic boundary conditions. The forcing function $f$ is determined by the requirment that $U$ is a solution of (26). We notice that no forcing is needed to ensure that $U$ is an exact solution for the inviscid case with $\eta=1$. We substitute a superposition of $U$ and a perturbation $v$,

$$
u(x, t)=U(x)+v(x, t)
$$

into (26) and linearize around $U$, omitting higher-order terms in $v$ :

$$
\partial_{t} v+(1-\eta) \sin (x) \partial_{x} v+\left(v+\eta \partial_{x}^{2} v\right) \cos (x)=v \partial_{x}^{2} v
$$

We use the following Fourier expansion for $v$ :

$$
v=\sum_{k=-\infty}^{\infty} \alpha_{k}(t) e^{i k x}
$$

where $i$ is the imaginary unit with $i^{2}=-1$. After substitution of this series into (28) and ordering of terms, we obtain an infinitie system of ordinary differential equations for the Fourier coefficients $\alpha_{k}$ :

$$
\dot{\alpha}_{k}=\frac{1}{2} k(\eta k-\eta-1) \alpha_{k-1}-k^{2} v \alpha_{k}+\frac{1}{2} k(\eta k+\eta+1) \alpha_{k+1},
$$

where $k$ is a number in $\mathbb{Z}$.

The instability encountered in the previous section concerns the gradient model, in which no eddyviscosity is adopted, while the molecular viscosity is relatively small. Therefore, to understand the nature of this instability for the Burgers equation, we first analyse system (30) assuming $v=0$. Instead of the infinite system, we consider a sequence of finite-dimensional systems,

$$
\dot{\mathbf{z}}_{n}=M_{n} \mathbf{z}_{n},
$$

where $\mathbf{z}_{n}$ is a vector containing the $2 n+1$ Fourier coefficients $a_{-n} \cdots a_{n}$ and $M_{n}$ is a $(2 n+1) \times(2 n+1)$ tridiagonal matrix:

$$
\mathbf{z}_{n}=\left[\begin{array}{l}
\alpha_{-n} \\
\vdots \\
\alpha_{-1} \\
\alpha_{0} \\
\alpha_{1} \\
\vdots \\
\alpha_{n}
\end{array}\right], \quad M_{n}=\left[\begin{array}{cccccccccc}
0 & l_{n} & & & & & & & \\
r_{n} & \ddots & \ddots & & & & & & \\
& \ddots & \ddots & l_{2} & & & & & \\
& & r_{2} & 0 & l_{1} & & & \\
& & & 0 & 0 & 0 & & \\
& & & & l_{1} & 0 & r_{2} & & \\
& & & & & l_{2} & \ddots & \ddots & \\
& & & & & & \ddots & \ddots & r_{n} \\
& & & & & & & l_{n} & 0
\end{array}\right],
$$

with

$$
\begin{aligned}
& l_{k}=\frac{1}{2} k(\eta k-\eta-1), \\
& r_{k}=\frac{1}{2}(k-1)(\eta k+1) .
\end{aligned}
$$

The eigenvalues of $A_{n}$ determine the stablity of the problem. The system is unstable if the maximum of the real parts of the eigenvalues is positive.

In the following we consider the asymptotic behavior for large values of $n$ of the maximum of the real parts of the eigenvalues of $M_{n}$. We denote the eigenvalues of $M_{n}$ by $\lambda_{i}$, whereas $\lambda_{\max }$ represents an eigenvalue with

$$
\left|\lambda_{\max }\right|=\max \left|\lambda_{i}\right|
$$


The proof of the following three properties is given in the Appendix:

1. If $\lambda$ is an eigenvalues, then $-\lambda$ is an eigenvalue.

2. $\left|\lambda_{\max }\right| \sim \eta n^{2}$.

3. $\left|\operatorname{Im}\left(\lambda_{\max }\right)\right| \leq n-1$.

The first point implies that $\lambda_{\max }$ can be chosen such that $\operatorname{Re}\left(\lambda_{\max }\right) \geq 0$. Hence, the combination of these three properties yields the asymptotic behavior of the maximum of the real parts of the eigenvalues:

$$
\operatorname{Re}\left(\lambda_{\max }\right) \sim \eta n^{2} .
$$

Thus we have shown that the inviscid system is linearly unstable and that the largest real part of the eigenvalues is asymptotically proportional to $n^{2}$, where $n$ is the number of Fourier modes taken into account.

It should be observed that the instability is severe, since the system is not only unstable, but the growth rate of the instability is infinitely large as $n \rightarrow \infty$. The instability is fully due to the incorporation of the gradient model, since all eigenvalues of the matrix $M_{n}$ are purely imaginary in case the inviscid Burgers equation without subgrid model is considered $(\eta=0)$. In numerical simulations the instability will grow with a finite speed, since then the number of Fourier modes is limited by the finite grid. Moreover, expression (39) illustrates that grid refinement (with $\eta$ kept constant), which corresponds to a larger $n$, will not stabilize the system, but only enhance the instability. In order to investigate whether three-dimensional simulations show features similar to this one-dimensional behavior, we have refined the grid of the unstable $32^{3}$ LES in the previous section to $64^{3}$ cells, using the same value for $\Delta$. Hence, whereas $\Delta=2 h$ in the simulation on the $32^{3}$ grid, $\Delta=4 h$ in the $64^{3}$ LES, where $h$ is the grid spacing. The $64^{3}$ LES with the gradient model collapsed at $t=21$, which is about a quarter of the collapse time of the corresponding $32^{3}$-LES model. However, if the filter width $\Delta$ is reduced simultaneously when the grid is refined, i.e., $\Delta / h$ is kept constant, the instability is not necessarily enhanced. This is illustated by a simulation on the $64^{3}$ grid using the gradient model with $\Delta=2 h$. This simulation collapsed at approximately the same time as the $32^{3}$ LES using $\Delta=2 h$. In fact the growth rate of the instability of the one-dimensional problem can be expressed in $\Delta$ and the grid spacing $h: \eta n^{2} \sim(\Delta / h)^{2}$. Consequently, the instability is not enhanced if the ratio between $\Delta$ and $h$ is kept constant.

Finally, we consider the more complicated case $v \neq 0$. The linear system in (30) now gives rise to matrices $M_{n}$ which have a negative principal diagonal. It is known that for every fixed value of $n$ there exists an eigenvalue arbitrarily close to the eigenvalue of the inviscid system $\left(\lambda_{\max }\right)$ if $v$ is sufficiently small (Chatelin, 1993 (Lemma 4.3.1)). Hence for small values of $v$ the viscous system for finite $n$ is still linearly unstable. The matrix $M_{n}$ is strictly diagonally dominant if

$$
v>\eta+1,
$$

while all rows except $n$ and $n+2$ are already diagonally dominant if $v>\eta$. If the matrix is diagonally dominant, the real parts of all eigenvalues are negative and, consequently, the system is stable. This indicates that stability can be achieved by a sufficiently large viscosity, which does not depend on $n$, but only on $\eta$. Thus, we conclude that if the gradient model is supplemented with an adequate eddy-viscosity the instability will be removed. The original Clark model, which indeed contains enough eddy-viscosity to avoid instabilities, is too dissipative. In the next section an adequate eddy-viscosity formulation which stabilizes the gradient model, while not being too dissipative, is presented for three-dimensional LES.

\section{The Dynamic Clark Model}

In the previous sections we have encountered two problems caused by the Clark model. If the Smagorinsky eddy-viscosity is incorporated, the model is too dissipative. However, if the eddy-viscosity part is omitted, the simulation becomes unstable. The analysis in the previous section indicated that this instability is caused by the model, not by the numerical method, and can be overcome by sufficient dissipation. In this section it is shown that the dynamic procedure is able to solve both problems. 
In order to overcome the excessive dissipation of the Smagorinsky model in laminar regimes the dynamic eddy-viscosity model has been developed by Germano (Germano, 1992) and has subsequently been applied to a number of flows (Moin and Jimenez, 1993). This model adopts Smagorinsky's eddy-viscosity formulation, but the square of the Smagorinsky constant is replaced by a coefficient which is dynamically obtained and depends on the local structure of the flow. Apart from the grid-filter level (" $F$-level"), denoted by the bar filter, Germano introduced a test filter (at the "G-level"), which is denoted by the hat $\left(^{\circ}\right)$ and corresponds to a filter width $2 \Delta$. The consecutive application of these two filters, resulting in, e.g., $\hat{\bar{\rho}}$, defines a filter on the " $F G$-level," with which a filter width $\kappa \Delta$ can be associated. For top-hat filters the optimum value for $\kappa$ equals $\sqrt{5}$ (Vreman et al., 1994b). Next we consider Germano's identity, which reads

$$
\hat{\rho} T_{i j}-\widehat{\bar{\rho} \tau_{i j}}=L_{i j}
$$

The right-hand side of (41) can be explicitly calculated from the variables on the $F$-level:

$$
L_{i j}=\left(\frac{\overline{\rho u}_{i} \overline{\rho u_{j}}}{\bar{\rho}}\right)-\frac{\widehat{\rho u_{i}} \widehat{\widehat{\rho u}}_{j}}{\hat{\bar{\rho}}},
$$

where $(\cdot)^{\wedge}$ indicates that the hat filter is applied to the expression between the brackets. This notation is used in conjunction with the identically defnied notation $\left(^{\circ}\right)$ for convenience in the exposure. The terms on the left-hand side of the Germano identity are the turbulent stress on the $F G$-level,

$$
\hat{\bar{\rho}} T_{i j}=\widehat{\overline{\rho u_{i} u_{j}}}-\frac{\widehat{\overline{\rho u}}_{i} \widehat{\overline{\rho u}}_{j}}{\hat{\bar{\rho}}},
$$

and the turbulent stress on the $F$-level filtered with the test filter, respectively. The terms on the left-hand side cannot be calculated from the variables on the $F$-level.

In order to obtain a dynamic eddy-viscosity model, Germano substituted the Smagorinsky eddyviscosity model into identity (41). Similarly, a dynamic mixed model has been formulated, which employs the mixed model by Bardina et al. as the base model (Zang et al., 1993; Vreman et al., 1994b). In the following we formulate the dynamic Clark model,

$$
\tilde{\rho} \tau_{i j}=\frac{1}{12} \Delta_{k}^{2} \bar{\rho} \partial_{k} \tilde{u}_{i} \partial_{k} \tilde{u}_{j}-C_{\mathrm{C}} \Delta^{2} \bar{\rho}|S(\tilde{\mathbf{u}})| S_{i j}(\tilde{\mathbf{u}}),
$$

which resembles (21), with the model constant $C_{\mathrm{S}}^{2}$ replaced by a model coefficient $C_{\mathrm{C}}$, which is allowed to be a function of space and time. In order to obtain an expression for $C_{\mathrm{C}}$, the subgrid model (equation (44)) is substituted into the Germano identity, which means that expressions for $T_{i j}$ and $\tau_{i j}$ are obtained by formulating the subgrid model in $F G$-filtered quantities and $F$-filtered quantities, respectively. Denoting the Favre-filtered velocity on the $F G$-level by

$$
v_{i}=\frac{\widehat{\overline{\rho u_{i}}}}{\widehat{\tilde{\rho}}}
$$

the Clark model on the $F G$-level reads

$$
\hat{\bar{\rho}} T_{i j}=\frac{1}{12}\left(\kappa \Delta_{k}\right)^{2} \hat{\bar{\rho}} \partial_{k} v_{i} \partial_{k} v_{j}-C_{\mathrm{C}}(\kappa \Delta)^{2} \hat{\bar{\rho}}|S(\mathbf{v})| S_{i j}(\mathbf{v}),
$$

where $|S(\mathbf{v})|^{2}=\frac{1}{2} S_{i j}^{2}(\mathbf{v})$. Substitution of (44) and (46) into the Germano identity (41) yields

$$
H_{i j}+C_{\mathrm{C}} M_{i j}=L_{i j}
$$

where $H_{i j}$ and $M_{i j}$ are terms related to the gradient and eddy-viscosity part of the Clark model, respectively:

$$
\begin{aligned}
& H_{i j}=\frac{1}{12}\left(\kappa \Delta_{k}\right)^{2} \hat{\bar{\rho}} \partial_{k} v_{i} \partial_{k} v_{j}-\left(\frac{1}{12} \Delta_{k}^{2} \bar{\rho} \partial_{k} \tilde{u}_{i} \partial_{k} \tilde{u}_{j}\right), \\
& M_{i j}=-\hat{\bar{\rho}}(\kappa \Delta)^{2}|S(\mathbf{v})| S_{i j}(\mathbf{v})+\left(\bar{\rho} \Delta^{2}|S(\tilde{\mathbf{u}})| S_{i j}(\tilde{\mathbf{u}})\right) \hat{.}
\end{aligned}
$$

To obtain the expression for $M_{i j}$ we have neglected variations of $C_{\mathrm{C}}$ on the scale of the test-filter width. Since, (47) represents a system of five equations for the single unknown $C_{C}$, a least-squares approach (Lilly, 
1992) is followed to calculate this coefficient,

$$
C_{\mathrm{C}}=\frac{\left\langle M_{i j}\left(L_{i j}-H_{i j}\right)\right\rangle}{\left\langle M_{i j} M_{i j}\right\rangle} .
$$

In order to prevent numerical instability, caused by negative values of $C_{C}$, the numerator and denominator in (50) are averaged over the homogeneous directions, which is expressed by the symbol $\langle\cdot\rangle$. In the simulations reported here the coefficient $C_{C}$ in the dynamic Clark model is artificially set to zero at locations where the right-hand side of (50) returns negative values. It has, however, been checked that simulations in which negative values of $C_{C}$ are allowed give rise to negligible differences.

This subgrid model satisfies the requirements following from the analysis in the previous section, if the coefficient $C_{\mathrm{C}}$ is sufficiently large. If on a given grid the model is stable, it is expected to remain stable after grid refinement. Indeed, the magnitude of the eddy-viscosity part of the dynamic Clark model, $C_{\mathrm{C}} \Delta^{2} \bar{\rho}|S(\tilde{\mathbf{u}})|$, varies only slightly with the mesh size when keeping $\Delta$ constant. Hence, this model enables a separation of numerical errors from modeling errors.

We compare results of the dynamic Clark model with the dynamic eddy-viscosity model (Germano, 1992) and the dynamic mixed model (Zang et al., 1993; Vreman et al., 1994b). The dynamic eddy-viscosity model employs the Smagorinsky model as the base model, and the dynamic model coefficient is obtained using (47) with the tensor $H_{i j}$ equal to zero. The dynamic mixed model employs the mixed model by Bardina et al. as the base model; in that case the tensor $H_{i j}$ in (47) is replaced by a tensor using the similarity model on different levels (Vreman et al., 1994b). With respect to computational efficiency the dynamic Clark model does not require much more work than the dynamic eddy-viscosity model, unlike the dynamic mixed model. The derivatives of the filtered velocity needed for the gradient part are already calculated in order to obtain the strain rate at different filter levels. The extra work needed is mainly due to the six filtering operations in (48) indicated by $(\cdot)^{\wedge}$. The calculation of $H_{i j}$ for the dynamic mixed model, however, contains considerably more filtering operations, required for the evaluation of the similarity model at different filtering levels. Consequently, the computational cost for the dynamic mixed model is considerably higher than for the dynamic eddy-viscosity and dynamic Clark model.

Figure 3 shows results of LES for the three dynamic models, using the same grid and filter as described in Section 3. Three quantities are compared: the evolution of the total kinetic energy $E$ and the momentum thickness $d$, and the profile of the turbulent kinetic energy $k$, which is defined as

$$
k\left(x_{2}\right)=\frac{1}{2}\left\langle\left(\tilde{u}_{i}-\left\langle\tilde{u}_{i}\right\rangle\right)^{2}\right\rangle .
$$

The $k$-profile is calculated at $t=70$, which is well beyond the starting point of the mixing transition process, but just before the final pairing has been accomplished. The time $t=70$ is rather arbitrary; it could equally well be $t=60$ or $t=80$, without altering the conclusions. All three models shows an improvement over the coarse-grid simulation without any subgrid model, indicating that in all three cases subgrid modeling makes sense. Furthermore, the results for the dynamic Clark and dynamic mixed model are much closer to the filtered DNS results than the results of the dynamic eddy-viscosity model, in particular for the evolution of the total kinetic energy and the momentum thickness.

Figure 4 shows the evolutions of the value of the dynamic model coefficient for the three dynamic models. As can be inferred from (50) the model coefficient is a function of time and the normal direction $x_{2}$ only. The results in Figure 4 show $C_{C}$ averaged over the $x_{2}$-direction. The dynamic eddy viscosity model gives rise to higher values for the model coefficient than the other two models. The reason is that the nonedddy viscosity parts of the dynamic Clark and dynamic mixed model already provide some dissipation and thus reduce the role of the eddy-viscosity part. Furthermore, it is observed that the similarity part in the dynamic mixed model is more dissipative than the gradient part in the dynamic Clark model, since the model coefficient is lower in the first case.

Hence, the dynamic Clark and dynamic mixed model lead to comparable simulation results, which are considerably more accurate than can be obtained with the dynamic eddy-viscosity model. Moreover, the dynamic procedure applied to the Clark model solves the problems encountered in Section 3. Firstly, the dynamic Clark model provides proper dissipation, thus avoiding the excessive dissipation of the original Clark model. On the other hand, the dissipation in the dynamic Clark model is sufficient to overcome the stability problem of the gradient model. We have verified that LES with the dynamic Clark model is also 


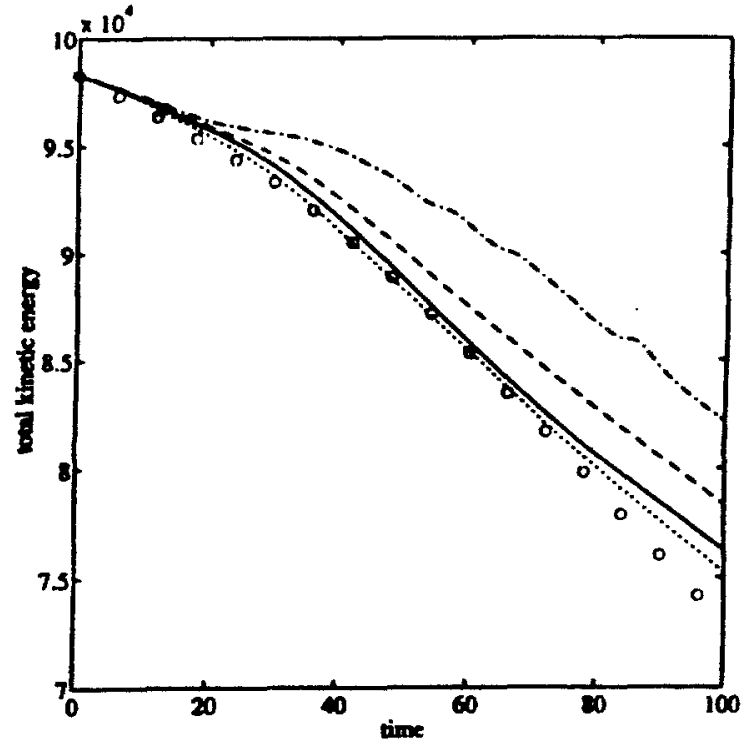

(a)

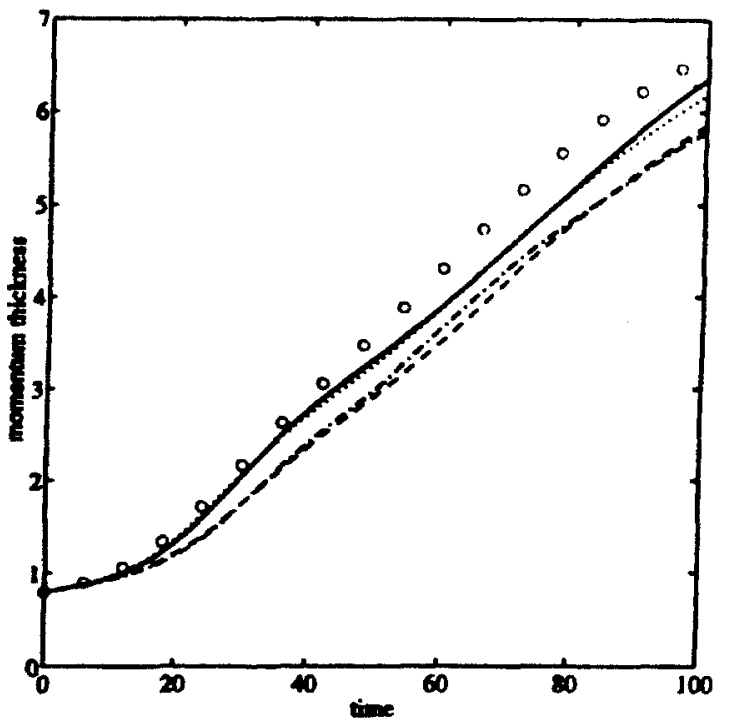

(b)

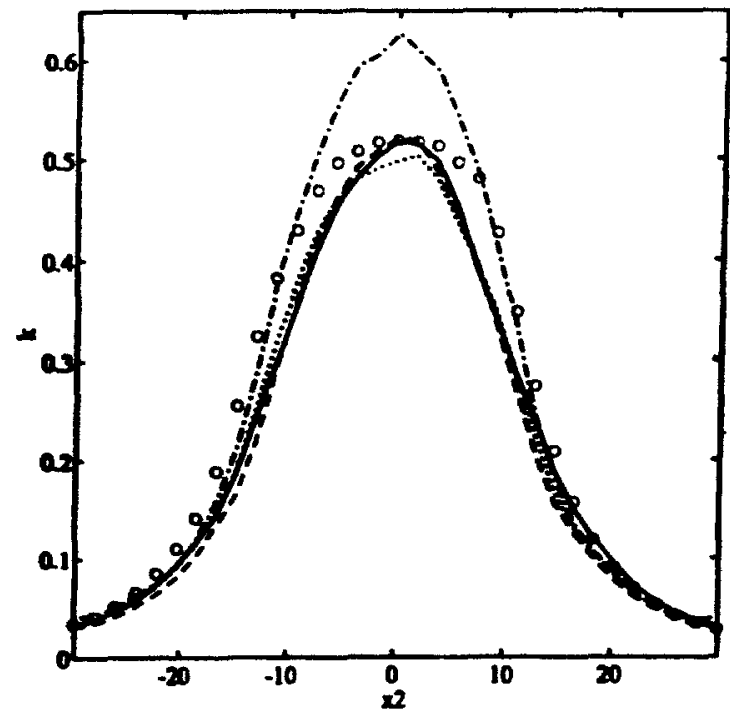

(c)

Figure 3. Results of LES with the dynamic Clark model (solid), the dynamic eddy-viscosity model (dashed), the dynamic mixed model (dotted), the filtered DNS (circles), and LES without a subgrid-model (dashed-dotted). The evolution of the total kinetic energy (a), the evolution of the momentum thickness (b), and the profile of the turbulent kinetic energy at $t=70$ (c) are shown.

stable at other grids, both for $\Delta=4 h$ keeping $\Delta$ constant, and for both $\Delta$ and $h$ taken smaller and retaining $\Delta=2 h$. These findings are in agreement with the analysis in Section 4 .

\section{Conclusions}

In this paper we have investigated the Clark subgrid-scale model, which is the sum of the gradient model, based on Taylor expansions, and the Smagorinsky eddy-viscosity model. We have extended the formal derivation of the incompressible Clark model to be able to use the model in compressible flow simulations as well. In LES of the weakly compressible mixing layer, the Clark model is too dissipative when the 
Figure 4. The averaged model coefficient for LES with the dynamic Clark model (solid), the dynamic eddy-viscosity model (dashed), and the dynamic mixed model (dotted).

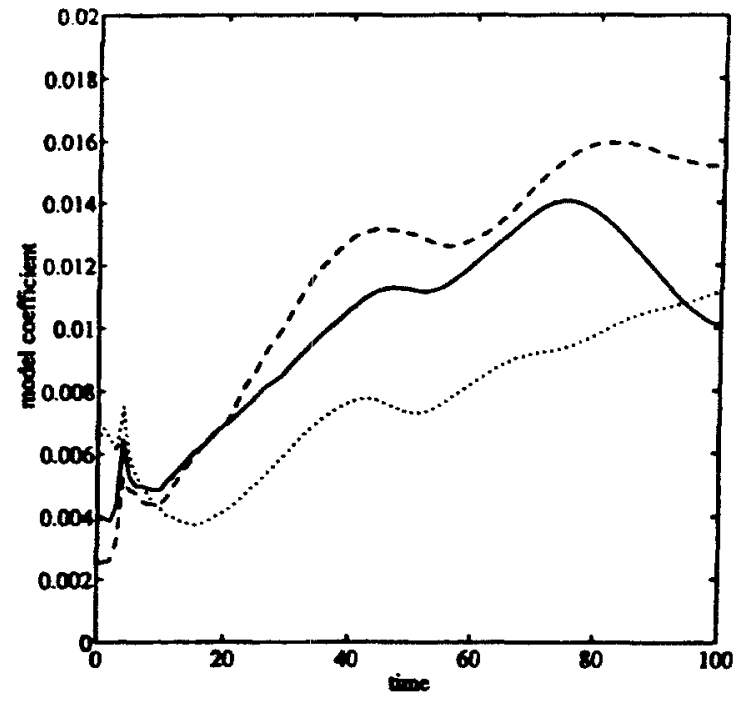

eddy-viscosity part is incorporated. However, the model destabilizes the simulation if the eddy-viscosity part is omitted. In order to analyse the gradient part of the Clark model and to clarify the nature of the instability, the one-dimensional Burgers equation supplemented with the gradient model has been considered. A linear stability analysis of a sinusoidal profile of the modified Burgers equation has been performed. In the limit of vanishing viscosity, the maximum of the positive real parts of the eigenvalues has been shown to be proportional to the square of the number of modes, if a finite number of modes is taken into account. This shows that the instability becomes more severe, if the resolution is increased. For an infinite number of modes, the growth-rate of the instability is infinitely large. These analytical results for the one-dimensional case are similar to the behavior of the instability observed in three-dimensional simulations with the gradient model. Furthermore, the one-dimensional analysis indicates that a sufficient amount of viscosity is able to solve the stability problem. For these reasons we have introduced the dynamic Clark model, which is the sum of the gradient model and a dynamic eddy-viscosity model. Simulations using this new model show that the dissipation is sufficient to prevent the instability encountered in simulations employing the pure gradient model. On the other hand the dynamic Clark model avoids the excessive dissipation in the transitional regime observed in simulations using the original Clark model.

The results obtained with the dynamic Clark model are reasonably accurate as comparison with filtered DNS results indicates. Furthermore, the dynamic Clark model has been compared with the standard dynamic eddy-viscosity model and the recently developed dynamic mixed model. Compared with the dynamic eddy-viscosity model, the dynamic Clark model gives more accurate predictions. Compared with the recently developed dynamic mixed model, the results are of comparable accuracy. With respect to computational costs the dynamic Clark model is more efficient than the dynamic mixed model, since considerably less extra filtering operations are required in order to evaluate the model. We conclude that the dynamic Clark model has been found to give good results for the weakly compressible temporal mixing layer. Understanding this model will further increase if it is also investigated in LES of different flows.

\section{Acknowledgments}

The authors are grateful to Ruud van Damme (University of Twente) for this contribution to the material in the appendix. Furthermore, the time for the computations was provided by the Stichting Nationale Computerfaciliteiten (National Computing Facilities Foundation, NCF), which is financially supported by the Nederlandse Organisatie van Wetenschappelijk Onderzoek (Netherlands Organization for Scientific Research, NWO). 


\section{Appendix}

In this appendix the proof of the three statements in (36)-(38) concerning the eigenvalues of the matrix $M_{n}$ is given. The structure of the matrix $M_{n}$ is such that an eigenvalue is equal to zero or an eigenvalue of the following matrix:

$$
A_{n}=\left[\begin{array}{ccccc}
0 & r_{2} & & & \\
l_{2} & \ddots & \ddots & & \\
& \ddots & \ddots & r_{n-1} & \\
& & l_{n-1} & 0 & r_{n} \\
& & & l_{n} & 0
\end{array}\right]
$$

Consequently, to consider the eigenvales of $A_{n}$ is sufficient. These eigenvalues are roots of the characteristic polynomial $P_{n}(\lambda)$ of the matrix $A_{n}$ :

$$
P_{n}(\lambda)=\operatorname{det}\left(\lambda I_{n}-A_{n}\right),
$$

where $I_{n}$ is the $n \times n$ unity matrix. If we decompose this determinant with respect to the last column, we obtain

$$
\begin{aligned}
& P_{1}(\lambda)=\lambda, \\
& P_{2}(\lambda)=\lambda^{2}-r_{2} l_{2}, \\
& P_{n}(\lambda)=\lambda P_{n-1}(\lambda)-r_{n} l_{n} P_{n-2}(\lambda), \quad n>2 .
\end{aligned}
$$

This recursive relation demonstrates that $P_{n}(\lambda)=0$ implies $P_{n}(-\lambda)=0$. Thus if $\lambda$ is an eigenvalue, $-\lambda$ is also an eigenvalue and thus property (36) has been established.

Next, we consider the statement about the asymptotic behavior of $\left|\lambda_{\max }\right|$. In order to prove this statement we formulate a lower and upper bound for $\left|\lambda_{\max }\right|$ which are both asymptotically proportional to $\eta n^{2}$. An upper bound is obtained when the Gershgorin theory is applied to $A_{n}$ :

$$
\left|\lambda_{\max }\right| \leq l_{n-1}+r_{n}=\eta(n-1)^{2} \sim \eta n^{2} .
$$

Since $\left|\lambda_{\max }\right|>\left|\lambda_{i}\right|$ for $i=1, \ldots, n$ and $\lambda_{i}$ represents one of the $n$ roots of the polynomial $P_{n}(\lambda)$, the following relation provides a lower bond for $\left|\lambda_{\max }\right|$ :

$$
\left|\lambda_{\max }\right| \geq\left|\lambda_{1} \lambda_{2} \cdots \lambda_{n}\right|^{1 / n}=\left|P_{n}(0)\right|^{1 / n} .
$$

The recursive relation (A.5) provides

$$
P_{n}(0)=-r_{n} l_{n} P_{n-2}(0),
$$

with

$$
r_{n} l_{n}=\frac{1}{4}(n-1) n\left(\eta^{2}\left(n^{2}-n\right)-\eta-1\right) \sim \frac{1}{4} \eta^{2}(n-1)^{2} n^{2} .
$$

If $n$ is even and the coefficient $l_{k}$ is nonzero for even $k\left(r_{k}\right.$ is always nonzero), we obtain the following estimate, using Stirling's formula:

$$
\left|\lambda_{\max }\right| \geq\left|P_{n}(0)\right|^{1 / n} \sim\left(\left(\frac{1}{4} \eta^{2}\right)^{n / 2}(n !)^{2}\right)^{1 / n} \geq \frac{1}{2} \eta\left(\frac{n}{e}\right)^{2} \sim \eta n^{2} .
$$

Equations (A.6) and (A.10) together yield

$$
\left|\lambda_{\text {max }}\right| \sim \eta n^{2}
$$

The case for odd $n$ is more complicated, but does not need to be considered, since the behavior for even $n$ already provides sufficient information about the system. Furthermore, the above argument alters when $l_{k}=0$ for a certain (even) value of $k$. If $l_{k}=0$, the characteristic polynomial can be written as

$$
P_{n}(\lambda)=P_{k+1}(\lambda) Q_{n-k+1}(\lambda),
$$


where $Q_{n-k+1}(\lambda)$ represents the characteristic polynomial of the tridiagonal matrix with lower diagonal $l_{k+1} \cdots l_{n}$ and upper diagonal $r_{k+1} \cdots r$. The maximum root of $Q_{n-k+1}$ can be estimated like in (A.10) and its asymptotic behavior is also proportional to $\eta n^{2}$.

Finally, we derive an upper bound for the imaginary part of an eigenvalue $\lambda$ of $A_{n}$. Denoting the corresponding eigenvector with $\mathbf{y}$ we have

$$
A_{n} \mathbf{y}=\lambda \mathbf{y} .
$$

The matrix $A_{n}$ can be split into a symmetric matrix $B_{n}$ and an antisymmetric matrix $C_{n}$ :

$$
A_{n}=B_{n}+C_{n}
$$

with

$$
B_{n}=\left[\begin{array}{cccc}
0 & b_{2} & & \\
b_{2} & \ddots & \ddots & \\
& \ddots & \ddots & b_{n} \\
& & b_{n} & 0
\end{array}\right], \quad C n=\left[\begin{array}{cccc}
0 & c_{2} & & \\
-c_{2} & \ddots & \ddots & \\
& \ddots & \ddots & c_{n} \\
& & -c_{n} & 0
\end{array}\right],
$$

and

$$
\begin{aligned}
& b_{k}=\frac{1}{2}\left(r_{k}+l_{k}\right)=\frac{1}{2} \eta k^{2}-\frac{1}{2} \eta k+\frac{1}{4}, \\
& c_{k}=\frac{1}{2}\left(r_{k}-l_{k}\right)=\frac{1}{2} k-\frac{1}{4} .
\end{aligned}
$$

In the following we denote the Euclidean inner product in $\mathbb{C}^{n}$ by $(\cdot, \cdot)$ and the complex conjugate by the superscript $*$. Next, we derive the following relation between $\lambda$ and $\lambda^{*}$ :

$$
\begin{aligned}
\lambda(\mathbf{y}, \mathbf{y}) & =\left(A_{n} \mathbf{y}, \mathbf{y}\right) \\
& =\left(B_{n} \mathbf{y}, \mathbf{y}\right)+\left(\mathbf{C}_{n} \mathbf{y}, \mathbf{y}\right) \\
& =\left(\mathbf{y}, B_{n}^{T} \mathbf{y}\right)+\left(\mathbf{y}, C_{n}^{T} \mathbf{y}\right) \\
& =\left(\mathbf{y}, B_{n} \mathbf{y}\right)-\left(\mathbf{y}, C_{n} \mathbf{y}\right) \\
& =\left(\mathbf{y}, A_{n} \mathbf{y}\right)-2\left(\mathbf{y}, C_{n} \mathbf{y}\right) \\
& =\lambda^{*}(\mathbf{y}, \mathbf{y})-2\left(\mathbf{y}, C_{n} \mathbf{y}\right) .
\end{aligned}
$$

Now we can express an upper bound for the imaginary part of $\lambda$ as

$$
\left|\operatorname{Im}(\lambda)=\frac{1}{2}\right| \lambda-\lambda^{*}|=| \frac{\left(\mathbf{y}, C_{n} \mathbf{y}\right)}{(\mathbf{y}, \mathbf{y})} \mid \leq \frac{\|\mathbf{y}\|\left\|C_{n} \mathbf{y}\right\|}{\|\mathbf{y}\|^{2}} \leq\|C\|_{\mathrm{sp}}
$$

since $\left\|C_{n} \mathbf{y}\right\| \leq\left\|C_{n}\right\|_{s \mathrm{p}}\|\mathbf{y}\|$, where the subscript sp denotes the spectral matrix norm. The spectral matrix norm $\|C\|_{\text {sp }}$ is defined as the square root of the maximum of the absolute eigenvalues of the matrix $C_{n}^{T} C_{n}$, which is equal to the maximum absolute eigenvalue of $C_{n}$, since $C_{n}$ is antisymmetric. Finally, the following result is obtained using the Gershgorin theory:

$$
|\operatorname{Im}(\lambda)| \leq\left|c_{n-1}\right|+\left|c_{n}\right|=n-1,
$$

which expresses an upper bound for the imaginary parts of the eigenvalues.

\section{References}

Bardina, J., Ferziger, J.H., and Reynolds, W.C. (1984). Improved turbulence models based on LES of homogeneous incompressible turbulent flows Report No. TF-19, Department of Mechanical Engineering, Stanford, CA.

Chatelin, F. (1993). Eigenvalues of Matrices. Wiley, Chichester.

Clark, R.A., Ferziger, J.H., and Reynolds, W.C. (1979). Evaluation of subgrid-scale models using an accurately simulated turbulent flow. J. Fluid Mech., 91, 1-16.

Comte, P., Lesieur, M., and Lamballais, E. (1992). Large- and small-scale stirring of vorticity and a passive scalar in a 3D temporal mixing layer. Phys. Fluids $A, \mathbf{4}, 2761$.

Erlebacher, G., Hussaini, M.Y., Speziale, C.G., and Zang, T.A. (1992). Toward the large-eddy simulations of compressible turbulent flows. J. Fluid Mech., 238, 155.

Favre, A. (1983). Turbulence: space-time statistical properties and behavior in supersonic flows. Phys. Fluids, 26, 2851-2863. 
Germano, M. (1992). Turbulence: the filtering approach. J. Fluid Mech., 238, 325-336.

Germano, M., Piomelli, U., Moin, P., and Cabot, W.H.(1991). A dynamic subgrid-scale eddy viscosity model. Phys. Fluids A, 3, 1760.

Henningson, D.S., and Reddy, S.C. (1994). On the role of linear mechanisms in transition to turbulence. Phys. Fluids, 6, 1396.

Humi, M. (1990). Optimal large eddy simulation in one dimension. Phys. Fluids A, 2, 1046.

Lilly, D.K. (1992). A proposed modification of the Germano subgrid-scale closure method. Phys. Fluids A, 4, 633.

Liu, S., Meneveau, C., and Katz, J. (1994). On the properties of similarity subgrid-scale models as deduced from measurements in a turbulent jet. $J$. Fluid Mech, to appear.

Love, M.D. (1980). Subgrid modelling studies with Burgers' equation. J. Fluid Mech., 100, 87.

Moin, P., and Jimenez, J. (1993). Large eddy simulation of complex turbulent flows. Proc. AIAA 24th Fluid Dynamics Conference, Orlando, FL.

Moser, R.D., and Rogers, M. (1993). The three-dimensional evolution of a plane mixing layer: pairing and transition to turbulence. J. Fluid Mech., 247, 275.

Piomelli, U., Zang, T. A., Speziale, C.G., and Hussaini, M.Y. (1990). On the large-eddy simulation of transitionl wall-bounded flows. Phys. Fluids A, 2, 257.

Ragab, S.A., and Wu, J.L. (1989). Liner instabilities in two-dimensional compressible mixing layers. Phys. Fluids A, 1, 957.

Rogallo, R.S., and Moin, P. (1984). Numerical simulation of turbulent flows. Ann. Rev. Fluid Mech., 16, 99-137.

Sandham, N.D., and Reynolds, W.C. (1991). Three-dimensional simulations of large eddies in the compressible mixing layer. $J$. Fluid Mech., 224, 133.

Schumann, U. (1991). Direct and large eddy simulation of turbulence-summar of the state-of-the-art 1991. Introduction to the Modeling of Turbulence, Lecture Series 1991-02, Von Karman Institute, Brussels.

Smagorinsky, J., (1963). General circulation experiments with the primitive equations. I. The basic experiment. Monthly Weather Rev., 91, 99-164.

Speziale, C.G. (1985). Galilean invariance of subgrid-scale stress models in the large eddy simulation of turbulence. J.Fluid Mech., 156, $55-62$.

Vreman, A.W., Geurts, B.J., Kuerten, J.G.M., and Zandbergen, P.J. (1992). A finite volume approach to large eddy simulation of compressible, homogeneous, isotropic, decaying turbulence. Internat. J. Numer Methods Fluids, 15, 799-816.

Vreman, B., Geurts, B., and Kuerten, H. (1993). A priori tests of large eddy simulation of the compressible plane mixing layer. Memorandum No. 1152, University of Twente, Enschede.

Vreman, B., Geurts, B., and Kuerten, H. (1994a). Realizability conditions for the turbulent stress tensor in large eddy simulation. J. Fluid Mech., 278, 351-362.

Vreman, B., Geurts, B., and Kuerten, H. (1994b). On the formulation of the dynamic mixed subgrid-scale model. Phys. Fluids, 6, 4057-4059.

Vreman, B., Geurts, B., and Kuerten, H. (1994c). Subgrid-modelling in LES of compressible flows. Proc. First ERFOCTAC Workshop on Direct and Large Eddy Simulation, Guildford.

Vreman, B., Geurts, B., and Kuerten, H. (1994d). Subgrid-modelling and numerical errors in the large eddy simulation of the temporal mixing layer. Memorandum No. 1208, University of Twente, Enschede.

Zang, Y., Street, R. L., and Koseff, J.R. (1993). A dynamic mixed subgrid-scale model and its application to turbulent recirculating flows. Phys. Fluids A, 5, 3186 . 